\title{
Laboratory evaluation of a specimen transport medium for downstream molecular processing of sputum samples to detect Mycobacterium tuberculosis
}

\section{Authors}

Shaheed V. Omar ${ }^{\# a, b}$, Remco P.H. Peters ${ }^{\mathrm{a}, \mathrm{c}}$, Nazir A. Ismail ${ }^{\mathrm{a}, \mathrm{b}}$, Andries W. Dreyer ${ }^{\mathrm{b}}$, Halima M. Said $^{\mathrm{b}}$, Thabisile Gwala ${ }^{\mathrm{b}}$, Nabila Ismail ${ }^{\mathrm{b}}$, P. Bernard Fourie ${ }^{\mathrm{a}}$

\author{
Affiliations \\ ${ }^{\mathrm{a}}$ Faculty of Health Sciences, Department of Medical Microbiology, University of Pretoria, Pretoria, South Africa \\ ${ }^{\mathrm{b}}$ Centre for Tuberculosis, National TB Reference Laboratory, National Institute for Communicable Diseases, \\ National Health Laboratory Service, Johannesburg, South Africa \\ ${ }^{\mathrm{c}}$ Anova Health Institute, Johannesburg, South Africa
}

Running Title

Transport medium for molecular detection of TB in sputum

\section{Corresponding author}

1 Modderfontein Road

Sandringham

Johannesburg

South Africa

Email: shaheedvo@nicd.ac.za.

\begin{abstract}
Background: Modern molecular-based approaches for the detection of M. tuberculosis in sputum samples promise quicker and more accurate detection of cases. However, processing sputum samples at central diagnostic facilities provides a diagnostic approach, but requires a safe and efficient system that is not affected by transport delays and ambient temperature to be feasible. We evaluated the technical properties of PrimeStore ${ }^{\circledR}$ - Molecular Transport Medium (PS-MTM) for its ability to inactivate mycobacteria, ensuring stability of DNA over time at ambient temperatures and to assess the compatibility of the transport medium with DNA extraction systems.
\end{abstract}


Methods: Assessment of the transport medium for application of sputum samples processed for the detection of $M$. tuberculosis included; the inactivation of $M$. tuberculosis in spiked sputum samples, compatibility of the medium with three commercial nucleic extraction systems and stability of DNA in the medium at ambient temperature over 28 days. We further performed a clinical laboratory evaluation on 256 sputum specimen sent for tuberculosis investigation.

Results: Complete inactivation of M. tuberculosis occurred within 30 minute of exposure at ratio of 1:3 for sputum to PS-MTM. Sputum specimen in PS-MTM showed very good compatibility with automated bead-based extraction systems, producing high DNA output (estimated lower limits of detection: $\sim 170 \mathrm{CFU} / \mathrm{mL}$ ). Furthermore, PS-MTM samples remained stable over 28 days at ambient temperature displaying no significant change over time in $\mathrm{Ct}$-values $(<5 \%$ on a mean starting value of 22.47). Of 256 clinical sputum specimens, $10.2 \%$ were culture positive and $11.0 \%$ positive by real-time PCR of PS-MTM samples.

Conclusions: Collecting and transporting sputum from TB suspects in PS-MTM offers safe transport at ambient temperature, DNA stability for extended periods without cooling and specimens directly suitable for molecular testing. This novel approach may support introduction and further scale-up of molecular diagnostics for TB in resource-limited settings.

\section{Keywords}

Mycobacterium tuberculosis; specimen transport medium; molecular detection; DNA stability

\section{INTRODUCTION}

Current tuberculosis (TB) infection rates are estimated to be the highest in the history of the disease, where 22 middle-to-low income countries are burdened with $80 \%$ of active disease (Paulson, 2013, WHO, 2010). To improve diagnosis of TB, the World Health Organization (WHO) promotes the early introduction of rapid molecular testing globally (WHO, 2013). In light of that, South Africa has replaced smear microscopy with the molecular Xpert MTB/RIF assay (Xpert, Cepheid, Sunnyvale, CA, USA) in its National algorithm. A similar scenario may not be possible in other countries due to relatively high associated cost and poor infrastructure. This warrants exploration of alternative approaches to introduce molecular diagnostics in high- 
burden countries, for example by transporting sputum specimens to a centralized molecular diagnostic facility. In that regard, safe (inactivated for purpose of infection control) and efficient (preservation and stability of DNA) transportation of sputum specimens is of paramount importance.

Several methods have been described for inactivation of viable Mycobacterium tuberculosis from cultures by either heat or a combination of heat and the use of chemicals (Djelouagji and Drancourt, 2006, Doig et al., 2002, Inoue et al., 2014). However, the only relevant published data on the effect of reagents added directly to sputum on the viability of TB bacilli is the sample reagent buffer (SR) of the Xpert assay. The SR has demonstrated capability of producing an 8 $\log$ reduction, within 15 minutes, in $M$. tuberculosis viability in sputum spiked with this organism (Helb et al., 2010). In M. tuberculosis-positive sputum specimens, with bacillary loads of less than $10^{7}$ colony forming units/milliliter $(\mathrm{CFU} / \mathrm{ml})$, total inactivation was achieved within 15 minutes (Banada et al., 2010). In practice, the SR buffer is only added to the sample when processed at the laboratory and not intended for specimen transport.

The effect of ambient transportation and subsequent delays to laboratory testing on sputum $M$. tuberculosis bacillary and DNA loads for molecular testing is not known, unlike the documented negative effects of delay on culture yield of sputum specimens (Banda et al., 2000, Paramasivan et al., 1983). However, studies on other pathogens in different clinical specimens have shown a negative effect on molecular testing when kept at ambient temperature for extended periods of time (Hasan et al., 2012, Ingersoll et al., 2008). In that context, the manufacturer of the Xpert assay recommends that sputum samples be kept for no longer than 3 days at ambient temperatures up to $35^{\circ} \mathrm{C}$ until processing (Banada, et al., 2010). Delays in transport and testing could negatively affect the diagnostic yield when using sputum specimens.

A liquid transport medium, PrimeStore ${ }^{\circledR}$ - Molecular Transport Medium (PS-MTM); Longhorn Vaccines \& Diagnostics, San Antonio, TX, USA) is a commercial specimen collection and transport solution, specifically formulated for downstream molecular diagnostic testing. The proposed use of this chemically defined medium, composed of chaotrophic, chelating, reducing and defoaming agents, detergents and electrolytes to buffer to an optimal $\mathrm{pH}$, is for inactivation and lysis of biological pathogens as well as stabilizing/preserving the released nucleic acids (DNA and RNA) for prolonged periods. PS-MTM has been reported to reduce viability of 
microorganisms almost completely (99.9\%) using a standard panel of variety of viruses, bacteria and fungi, but not including M. tuberculosis (Daum et al., 2011). Furthermore, complete viral inactivation of enveloped viruses occurred within seconds after being placed in PS-MTM and viral RNA was preserved for up to 30 days at ambient temperature (Daum, et al., 2011).

Expanding potential use of PS-MTM to TB diagnostics, a recent study of PS-MTM showed inactivation within $30 \mathrm{~min}$ of $M$. tuberculosis at a bacillary load of $10^{6} \mathrm{CFU} / \mathrm{ml}$ (Daum et al., 2014). Based on the relatively scant information on the use of PS-MTM for TB diagnosis and research, as indicated above, insight into its potential application in routine diagnostics of TB is warranted. In this study, we aim to determine further the performance and safety potential use of PS-MTM as a collection vehicle and transport preservation method of sputum specimens for molecular detection and characterization of M. tuberculosis.

\section{MATERIALS AND METHODS}

\subsection{Design and steps of evaluation}

The value of PS-MTM was determined in several steps: (a) potential for inactivation of $M$. tuberculosis at high bacterial loads with both cultured bacilli as well as spiked sputum samples, (b) effect on preservation of $M$. tuberculosis DNA over time, (c) compatibility with various downstream nucleic acid extractions systems, (d) use of swabs to inoculate sputum into PSMTM tubes, (e) laboratory diagnostic performance of this system on clinical sputum specimens. Ethics approval for this study was obtained from the University of Pretoria's Research Ethics Committee of the Faculty of Health Sciences (129/2010).

\subsection{Preparation of $M$. tuberculosis suspensions for laboratory evaluations}

Standard M. tuberculosis suspensions were prepared by growing the M. tuberculosis H37Rv laboratory strain (ATCC 27294) as per routine protocol to the turbidity of a 0.5 McFarland standard; the equivalent concentration of $4.5 \times 10^{8} \mathrm{CFU} / \mathrm{ml}$ (Bollela et al., 1999, Global Laboratory Initiative, 2014, Murray and Baron, 2007). The optical turbidity was measured using the PhoenixSpec Nephelometer (BD Diagnostics, Franklin lakes, NJ, USA) against a standard curve. A series of ten-fold dilutions was prepared from the McFarland standard, producing 
concentrations ranging from $10^{6}$ to $10^{1} \mathrm{CFU} / \mathrm{ml}$. Manual colony counts to confirm the dilution series plated on Middlebrook 7H11 agar medium were performed for the concentrations that could be enumerated $\left(10^{1}-10^{3} \mathrm{CFU} / \mathrm{ml}\right)$.

\subsection{Inactivation of M. tuberculosis in PS-MTM}

The ability of PS-MTM to inactivate M. tuberculosis was assessed for purified culture isolates and spiked sputum samples. A $0.5 \mathrm{ml}$ suspension of M. tuberculosis $\mathrm{H} 37 \mathrm{Rv}$ strain $(\sim 1.5 \mathrm{x}$ $10^{8} \mathrm{cfu} / \mathrm{ml}$ ) was inoculated into $1.5 \mathrm{ml}$ of PS-MTM, briefly vortexed and sampled after ambient temperature incubation for 5, 10, 20, 40, 80 and 160 seconds. Sample aliquots of 0.2ml were directly plated on Middlebrook 7H11 agar and 0.5ml inoculated into liquid culture and processed using the Bactec Mycobacterial Growth Indicator Tubes (MGIT) 960 TM (Becton Dickinson Diagnostics, Sparks, MD, USA) as previously described (Siddiqi and Rusch-Gerdes, 2006). A positive control was included (suspension in saline without PS-MTM) and the experiment was

performed in triplicate. Sputum samples not submitted for TB investigation were obtained from the routine diagnostic laboratory at the University of Pretoria and assessed for the presence of acid-fast bacilli (AFB) by smear microscopy, cultured by MGIT to confirm the absence of $M$. tuberculosis followed by quality assessment using the Bartlett Scoring System (Winn Jr et al., 2005). Good quality purulent sputum specimens (Bartlett test score of 2+) were included for use in the spiking matrix experiments. These sputa were split and spiked with M. tuberculosis H37Rv strain with concentrations of $1.5 \times 10^{6}$ and $1.5 \times 10^{8} \mathrm{CFU} / \mathrm{ml}$ followed by inoculation into PS-MTM (without decontamination or other pre-culture steps). A matrix assessment to determine effect of concentration was performed by adding to $1 \mathrm{ml}$ of spiked sputum $3,2,1,0.5$ and $0.2 \mathrm{ml}$ of PS-MTM. Samples were incubated in triplicate at ambient temperature for 1, 5, 10, 30, 60 and 180 minutes, including two positive and negative controls, and analyzed using the MGIT 960 system. Effective inactivation at certain concentration and time point was defined as no growth in all samples after 42 days.

\subsection{Evaluation of DNA stability in sputum inoculated in PS-MTM}

Three sputum specimens, positive for M. tuberculosis on microscopy for AFB, with a smear microscopy grading of $3+$ were included in this evaluation. The sputum specimens were split into two equal aliquots with one added to PS-MTM at 1:2, and one refrigerated, with sterile 
water added to the latter aliquots at the same ratio as PS-MTM. Nucleic acid extraction using NucliSENS easyMAG was performed on all test samples at baseline and weekly intervals over a 4 week period. This time period would be sufficient for the primary diagnosis, extended delays due to logistic challenges in countries with poor infrastructure and if needed specimen availability for additional reflex molecular testing if required. Preservation of DNA was measured by real-time PCR on each of the extracts where an absence of significant decrease in cycle-threshold (CT) scores over tested time points indicates stability.

\subsection{Compatibility with three DNA extraction systems}

Ten-fold dilutions of the M. tuberculosis stock solutions were prepared to a range of $10^{1}$ to $10^{6} \mathrm{CFU} / \mathrm{ml}$ and spiked into 20 remnant clinical culture-negative sputum samples (as described in 2.2 and 2.3). These sputum samples were inoculated into PS-MTM at a volume ratio of 1:2. The final concentration of M. tuberculosis in the spiked sputum ranged from an estimated 3 to $250000 \mathrm{CFU} / \mathrm{ml}$. Aliquots of sputum in PS-MTM were prepared and processed using the following DNA extraction systems as per manufacturers' instructions: the QiaAMP DNA mini kit (Qiagen, Hilden, Germany), the MagNA Pure 96 System (Roche Diagnostics, Mannheim, Germany) using the DNA Bacterial/Viral small volume kit, the NucliSENS easyMAG (Biomerieux, Marcy I'Etoile, France) using the generic protocol. The all-in-one extraction and detection system, the Xpert MTB/Rif assay was performed as a control for routine detection; an

aliquot of sputum sample was tested by Xpert before inoculating in PS-MTM, as instructed by the manufacturer (Fig. 1). An input volume of $200 \mu$ l PS-MTM sample was used with $50 \mu 1$ output volume except in the case of MagNA Pure 96 (Roche, Germany) where the final volume was $100 \mu 1$. Real-time PCR (Daum et al., 2011) targeting the M. tuberculosis specific insertion sequence element 6110 (IS6110) was used on the LightCycler 480II platform (Roche Diagnostics, Mannheim, Germany) to detect M. tuberculosis from DNA extracts. 
Figure 1. Experimental design for assessment of compatibility of PrimeStore ${ }^{\circledR}$ - Molecular Transport Medium with different molecular platforms

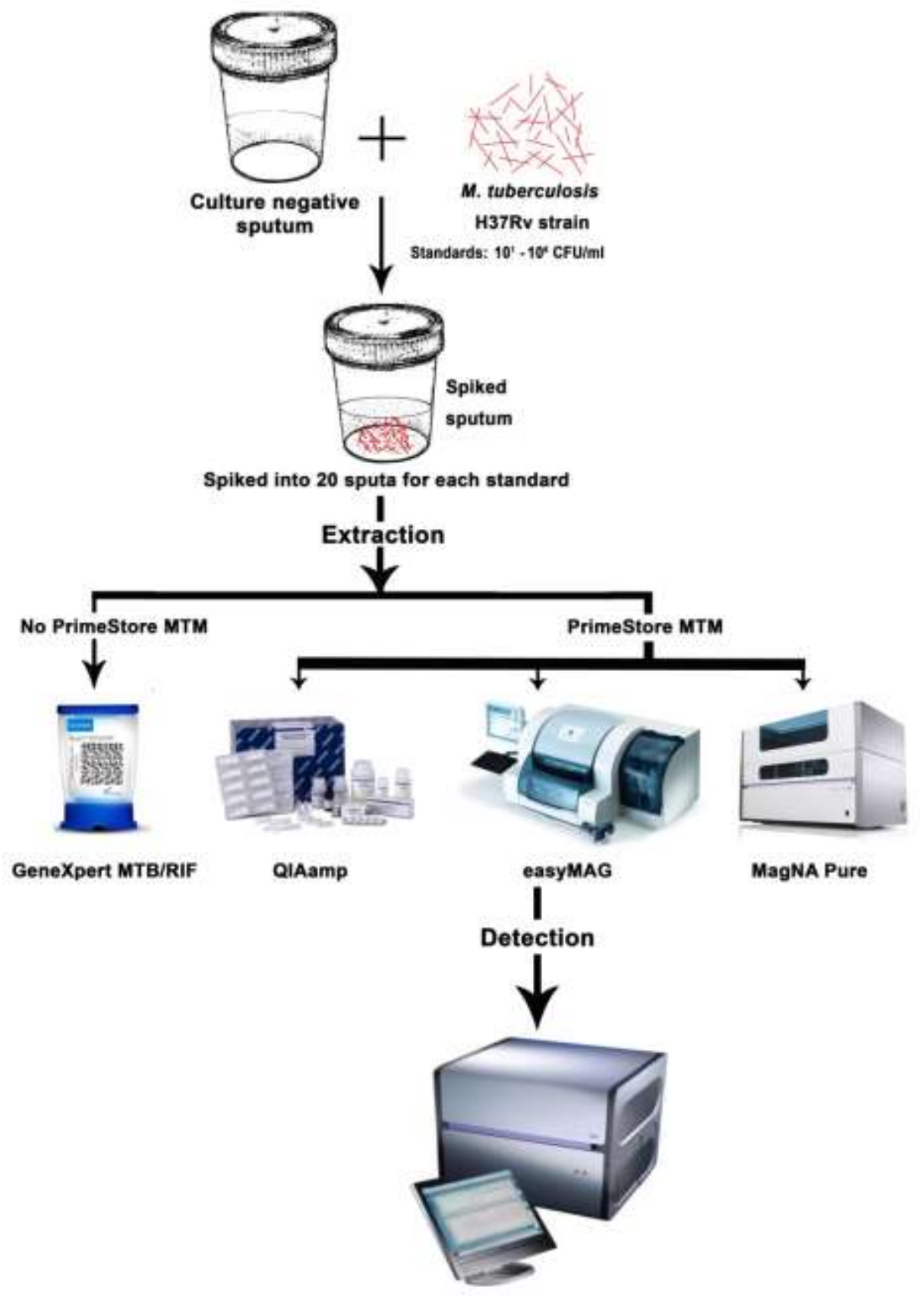




\subsection{Evaluation of using swabs to inoculate sputum into PS-MTM for molecular testing}

Flocculated cotton swabs (Copan Diagnostics Inc., Brescia, Italy) were evaluated for capacity to inoculate sputum into PS-MTM for downstream molecular testing. This swab-for-inoculation procedure was further evaluated in fourteen sputum specimens that were culture positive for $M$. tuberculosis and with a known smear microscopy grading for AFB. The volume captured on swab was determined by the difference in swab weight pre- and post- collection using a calibrated laboratory scale (Adam Equipment Co. Ltd, Milton Keynes, UK). As a control the remaining volume was pipetted into an equal volume of PS-MTM. After inoculation, PS-MTM tubes were kept overnight at ambient temperature followed by DNA extraction on the MagNA Pure 96 System and real-time PCR by LightCycler 480II.

\subsection{Evaluation of detecting M. tuberculosis in clinical samples stored in PS-MTM}

A total of 297 sputum specimens sent for TB investigation were prospectively collected at the Tshwane Academic Division (University of Pretoria) of the National Health Laboratory Service, South Africa. Specimens were included irrespective of quality; however, a minimum volume of $2 \mathrm{ml}$ was required to prevent compromising the routine testing. From each specimen, an aliquot was transferred into PS-MTM using the swab procedure. Routine diagnostic testing of specimens was performed using fluorescent microscopy (Auramine-O), mycobacterial culture on the MGIT 960 system with confirmation of $M$. tuberculosis in positive cultures by the TBcID rapid antigen test (BD, Sparks, MD, USA). DNA was extracted from the specimens stored in PS-MTM using the MagNA Pure 96 System followed by real-time PCR for M. tuberculosis detection by LightCycler 480II.

\section{RESULTS}

\subsection{Inactivation of M. tuberculosis in PS-MTM}

In order to assess the inactivation ability of PS-MTM, the high concentration suspension (of the reference strain was inoculated in triplicate into PS-MTM, stored at ambient temperature and followed by sampling after different interaction times ( $\min .5$ to max. 60 seconds). There was no growth of M. tuberculosis after 42-day incubation period in the MGIT 960 liquid culture system 
and on solid media compared to the positive control where growth was observed after 11 and 14 days respectively in the different culture systems.

Smear negative sputa with a positive Bartlett score were spiked with M. tuberculosis concentration of $1.5 \times 10^{6} \mathrm{CFU} / \mathrm{ml}$ showed no growth after 42 days at the ratios of 1:1, 1:2 and 1:3 for sputum vs. PS-MTM, except for one specimen at the one minute interval (Tab.1). The latter showed growth in liquid culture after 24 hours, but a sub-culture of this same sample (on Middlebrook 7H10 agar) showed no mycobacterial growth. Full growth of M. tuberculosis was observed when the volume of sputum exceeds the volume of PS-MTM (2:1 and 5:1). Erratic growth was observed at each time point at the higher inoculation concentration $\left(1.5 \times 10^{8}\right.$ $\mathrm{CFU} / \mathrm{ml}$ ) with a 1:1 ratio. An exposure time of 60 minutes was required for inactivation of $M$. tuberculosis with a ratio of at least 1:2 for sputum vs. PS-MTM.

Table1. Culture detection of $M$. tuberculosis from sputum samples spiked into PrimeStore® - Molecular Transport Medium at varying concentration, volume ratio and exposure time

\begin{tabular}{|c|c|c|c|c|c|c|c|c|}
\hline \multirow{2}{*}{$\begin{array}{l}\text { Concentration } \\
\text { of } M . \\
\text { tuberculosis } \\
\text { spiked in } \\
\text { sputum }\end{array}$} & \multirow{2}{*}{$\begin{array}{l}\text { Ratio of } \\
\text { spiked } \\
\text { sputum } \\
\text { to } \\
\text { PS- } \\
\text { MTM }\end{array}$} & \multicolumn{7}{|c|}{ Exposure time (minutes) } \\
\hline & & 1 & 5 & 10 & 15 & 30 & 60 & 180 \\
\hline \multirow{5}{*}{$1.5 \times 10^{6} \mathrm{CFU} / \mathrm{ml}$} & $0.33: 1$ & 0 & 0 & 0 & 0 & 0 & 0 & 0 \\
\hline & $0.5: 1$ & $1 / 3$ & 0 & 0 & 0 & 0 & 0 & 0 \\
\hline & 01:01 & 0 & 0 & 0 & 0 & 0 & 0 & 0 \\
\hline & 02:01 & $3 / 3$ & $3 / 3$ & $3 / 3$ & $3 / 3$ & $3 / 3$ & $3 / 3$ & $3 / 3$ \\
\hline & 05:01 & $3 / 3$ & $3 / 3$ & $3 / 3$ & $3 / 3$ & $3 / 3$ & $3 / 3$ & $3 / 3$ \\
\hline \multirow{5}{*}{$1.5 \times 10^{8} \mathrm{CFU} / \mathrm{ml}$} & $0.33: 1$ & 0 & $1 / 3$ & 0 & $1 / 3$ & 0 & 0 & 0 \\
\hline & $0.5: 1$ & $3 / 3$ & 0 & 0 & 0 & $2 / 3$ & 0 & 0 \\
\hline & 01:01 & $3 / 3$ & $1 / 3$ & $2 / 3$ & 0 & 0 & $1 / 3$ & $2 / 3$ \\
\hline & 02:01 & $3 / 3$ & $3 / 3$ & $3 / 3$ & $3 / 3$ & $3 / 3$ & $3 / 3$ & $3 / 3$ \\
\hline & 05:01 & $3 / 3$ & $3 / 3$ & $3 / 3$ & $3 / 3$ & $3 / 3$ & $3 / 3$ & $3 / 3$ \\
\hline
\end{tabular}

\subsection{Evaluation of DNA stability in PS-MTM}

Three microscopy smear-positive samples were split into two aliquots and stored in either PSMTM at ambient temperature or in a normal sputum container, without processing, at 
refrigeration temperature $\left(4^{\circ} \mathrm{C}\right)$. PCR detection of $M$. tuberculosis DNA from these containers was done on weekly basis for a period of four weeks. There was no significant change in Ctvalue $(<5 \%)$ over time observed for samples stored in PS-MTM or for those stored at $4^{\circ} \mathrm{C}$ in a normal sputum container (Tab. 2).

Table 2: Cycle threshold values of real-time PCR of M. tuberculosis DNA from sputum samples kept over a 28-day period in PrimeStore $\AA$ - Molecular Transport Medium (PS-MTM) at ambient temperature compared to storage in a normal sputum container at refrigeration temperature $\left(4^{\circ} \mathrm{C}\right)$.

\begin{tabular}{|c|ccc|ccc|}
\hline & \multicolumn{3}{|c|}{$\begin{array}{c}\text { Sputum in PS-MTM } \\
\text { Ambient temperature }\end{array}$} & \multicolumn{3}{c|}{ Sputum only4 ${ }^{\circ}$ C } \\
\hline Day(s) & Sample1 & Sample2 & Sample3 & Sample1 & Sample2 & Sample3 \\
\hline 0 & 22.95 & 23.48 & 20.97 & 22.84 & 23.09 & 21.80 \\
7 & 22.54 & 23.07 & 20.58 & 22.50 & 22.96 & 20.64 \\
14 & 22.58 & 23.23 & 20.41 & 22.85 & 23.38 & 20.97 \\
21 & 22.87 & 23.47 & 20.97 & 23.39 & 23.60 & 21.72 \\
28 & 22.49 & 22.99 & 20.57 & 22.65 & 23.56 & 20.97 \\
\hline
\end{tabular}

Note. Ambient temperature in this setting is generally $25-30^{\circ} \mathrm{C}$.

\subsection{Compatibility with DNA extraction systems}

The automated magnetic bead-based extraction systems (NucliSENS EasyMAG and MagNA Pure 96 System) had estimated lower limits of detection of 169 and $173 \mathrm{CFU} / \mathrm{ml}$ respectively producing the highest DNA yield from specimens stored in PS-MTM (Tab.3). The yield was higher than for the silica-based extraction system (QiaAMP) which detected all replicates up to the $25000 \mathrm{CFU} / \mathrm{ml}$ concentration, but missed an increasing proportion of replicates at subsequent (lower) concentrations (2,500-25,000 CFU/ml) that were not missed by the bead-based methods (Tab.3). A significant difference was seen between the MagNA Pure and positive control (Xpert system) in their ability to detect positives a various concentration, with MagNA Pure maintaining 
Table 3: Performance of DNA extraction systems by real-time PCR detection ofspiked sputum samples with varying concentrations of $M$. tuberculosis stored in PrimeStore ${ }^{\circledR}$ - Molecular Transport Medium (PS-MTM). The number of replicates detected and mean CT scores for those detected are indicated

\begin{tabular}{|c|c|c|c|c|c|c|c|c|c|c|c|c|c|}
\hline & & \multicolumn{12}{|c|}{ Mycobacterium tuberculosis CFU/ml in sputum } \\
\hline & & \multicolumn{2}{|c|}{250,000} & \multicolumn{2}{|c|}{25,000} & \multicolumn{2}{|c|}{2,500} & \multicolumn{2}{|c|}{250} & \multicolumn{2}{|c|}{25} & \multicolumn{2}{|c|}{3} \\
\hline System* & $\begin{array}{c}\text { Lower limit of } \\
\text { detection } \\
\text { (CFU/ml) [CI } \\
95 \%]\end{array}$ & $\begin{array}{c}\text { No. of } \\
\text { positive } \\
\text { replicates }\end{array}$ & $\begin{array}{c}\text { Mean } \\
\text { CT } \\
\text { score } \\
\text { (SD) }\end{array}$ & $\begin{array}{c}\text { No. of } \\
\text { positive } \\
\text { replicates }\end{array}$ & $\begin{array}{c}\text { Mean CT } \\
\text { score } \\
\text { (SD) }\end{array}$ & $\begin{array}{c}\text { No. of } \\
\text { positive } \\
\text { replicates }\end{array}$ & $\begin{array}{c}\text { Mean CT } \\
\text { score } \\
\text { (SD) }\end{array}$ & $\begin{array}{c}\text { No. of } \\
\text { positive } \\
\text { replicates }\end{array}$ & $\begin{array}{c}\text { Mean CT } \\
\text { score } \\
\text { (SD) }\end{array}$ & $\begin{array}{c}\text { No. of } \\
\text { positive } \\
\text { replicates }\end{array}$ & $\begin{array}{c}\text { Mean CT } \\
\text { score } \\
\text { (SD) }\end{array}$ & $\begin{array}{c}\text { No. of } \\
\text { positive } \\
\text { replicates }\end{array}$ & $\begin{array}{c}\text { Mean CT } \\
\text { score } \\
\text { (SD) }\end{array}$ \\
\hline & & \multicolumn{2}{|c|}{$(\mathbf{n}=20)$} & \multicolumn{2}{|c|}{$(\mathrm{n}=20)$} & \multicolumn{2}{|c|}{$(\mathbf{n}=\mathbf{2 0})$} & \multicolumn{2}{|c|}{$(\mathrm{n}=20)$} & \multicolumn{2}{|c|}{$(\mathbf{n}=\mathbf{2 0})$} & \multicolumn{2}{|c|}{$(\mathbf{n}=\mathbf{2 0})$} \\
\hline $\begin{array}{c}\text { MagNA } \\
\text { Pure } \dagger\end{array}$ & $\begin{array}{c}169 \\
{[62-1,698]}\end{array}$ & 20 & $\begin{array}{l}26.33 \\
(0.97)\end{array}$ & 20 & $\begin{array}{l}29.96 \\
(1.62)\end{array}$ & 20 & $\begin{array}{l}33.19 \\
(0.75)\end{array}$ & 20 & $\begin{array}{l}33.45 \\
(2.63)\end{array}$ & 13 & $\begin{array}{l}34.71 \\
(0.46)\end{array}$ & 7 & $\begin{array}{l}34.68 \\
(0.68)\end{array}$ \\
\hline EasyMAG & $\begin{array}{c}173 \\
{[78-891]}\end{array}$ & 20 & $\begin{array}{l}25.13 \\
(0.59)\end{array}$ & 20 & $\begin{array}{l}29.32 \\
(2.13)\end{array}$ & 20 & $\begin{array}{l}32.19 \\
(0.99)\end{array}$ & 20 & $\begin{array}{l}34.09 \\
(2.98)\end{array}$ & 10 & $35(0)$ & 2 & $35(0)$ \\
\hline QiaAMP & $\begin{array}{c}6397 \\
{[3,162-53,703]} \\
{[1,660-74,131]}\end{array}$ & 20 & $\begin{array}{l}25.64 \\
(2.62)\end{array}$ & 20 & $\begin{array}{l}29.40 \\
(2.03)\end{array}$ & 16 & $\begin{array}{l}32.20 \\
(1.36)\end{array}$ & 18 & $\begin{array}{l}34.22 \\
(2.81)\end{array}$ & 7 & $\begin{array}{l}37.00 \\
(1.57)\end{array}$ & 4 & $\begin{array}{c}35.7 \\
(1.40)\end{array}$ \\
\hline $\begin{array}{c}\text { Positive } \\
\text { control (Xpert } \\
\text { MTB/RIF } \\
\text { assay) } \dagger\end{array}$ & $\begin{array}{c}8749 \\
\\
{[1,660-} \\
74,131]\end{array}$ & 20 & $\begin{array}{l}23.51 \\
(1.50)\end{array}$ & 20 & $\begin{array}{l}26.71 \\
(2.23)\end{array}$ & 15 & $\begin{array}{l}29.20 \\
(3.53)\end{array}$ & 5 & $\begin{array}{l}29.66 \\
(5.24)\end{array}$ & 3 & $\begin{array}{l}27.83 \\
(4.51)\end{array}$ & $\mathbf{0}$ & - \\
\hline
\end{tabular}

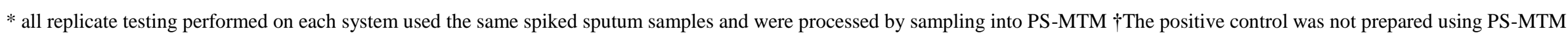


its ability to detect positives at the lower $\mathrm{CFU} / \mathrm{ml}$ concentration (Chi-square $=15.23 ; \mathrm{p}=0.009$ ). None of the other systems produced significantly different results from the Xpert control.

\subsection{Swab procedure for inoculation of sputum into PS-MTM tubes}

Using clinical culture-positive specimens, the estimated median volume of sputum captured by the swabbing procedure was $50 \mu 1$ and ranged from $50 \mu 1-250 \mu 1$ where samples with a higher

Table 4:Performance of swabbing procedure for transferring sputum into PrimeStore ${ }^{\circledR}$ - Molecular Transport Medium (PS-MTM) for the detection of M. tuberculosis in fourteen culture-positive samples with range of smear microscopy grades

\begin{tabular}{|c|c|c|c|c|c|c|c|}
\hline & $\begin{array}{l}\text { Sample } \\
\text { number }\end{array}$ & Viscosity & $\begin{array}{c}\text { Smear } \\
\text { Microscopy } \\
\text { grade* }\end{array}$ & $\begin{array}{c}\text { Captured } \\
\text { sputum } \\
\text { Volume (ml) }\end{array}$ & $\begin{array}{c}\text { Volume ratio of } \\
\text { Swabbed sputum } \\
\text { in PS-MTM }\end{array}$ & $\begin{array}{c}\text { Ct-value of PCR } \\
\text { after swab } \\
\text { inoculation }^{¥}\end{array}$ & $\begin{array}{c}\text { Ct-value of } \\
\text { PCR after } \\
\text { pipette } \\
\text { inoculation } * * *\end{array}$ \\
\hline \multirow{7}{*}{ 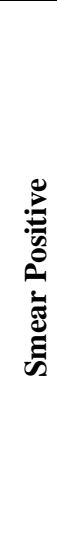 } & 1 & Purulent & +++ & 0.05 & $1: 30$ & 32.74 & 28.96 \\
\hline & 2 & Purulent & +++ & 0.05 & $1: 30$ & 26.56 & 23.97 \\
\hline & 3 & Salivary & ++ & 0.15 & $1: 10$ & 28.96 & 26.30 \\
\hline & 4 & Salivary & + & 0.25 & $1: 6$ & 31.67 & 35.00 \\
\hline & 5 & Purulent & scanty 9 & 0.05 & $1: 30$ & 33.75 & 31.76 \\
\hline & 6 & Purulent & scanty 7 & 0.05 & $1: 30$ & 31.85 & 30.20 \\
\hline & 7 & Purulent & scanty 1 & 0.05 & $1: 30$ & 34.21 & 33.59 \\
\hline \multirow{7}{*}{ 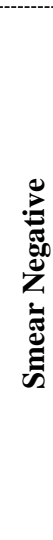 } & 8 & Purulent & Negative & 0.05 & $1: 30$ & 33.89 & 35.00 \\
\hline & 9 & Purulent & Negative & 0.05 & $1: 30$ & 32.72 & 32.68 \\
\hline & 10 & Salivary & Negative & 0.1 & $1: 15$ & 29.19 & 26.16 \\
\hline & 11 & Purulent & Negative & 0.05 & $1: 30$ & 34.47 & Negative \\
\hline & 12 & Purulent & Negative & 0.05 & $1: 30$ & 33.59 & 30.05 \\
\hline & 13 & Salivary & Negative & 0.1 & $1: 15$ & 33.11 & 35.00 \\
\hline & 14 & Salivary & Negative & 0.1 & $1: 15$ & Negative & Negative \\
\hline
\end{tabular}

*WHO smear microscopy scoring system: For 3 scanty samples, numbers 9, 7, 1 indicate AFB/100 FOV

**Sputum was inoculated at 1:1 ratio in PrimeStore (equal volume)

${ }^{\ddagger}$ No statistically significant differences across all results (smear positive and negative) between swab and pipette inoculation (Paired T $=0.70, \mathrm{DF}=13, \mathrm{p}=0.50)$. 
viscosity (purulent) retained a lower volume of sample compared to those less viscous (salivary) (Tab.4).M. tuberculosis DNA was detected by real-time PCR in all samples using the swabbing procedure and in all except one of the pipetted samples. Cycle threshold values were generally lower for pipetted specimens, but the swab to PS-MTM ratio was relatively higher for those samples (1:1). DNA concentrations after extraction ranged between 231 and $281 \mathrm{ng} / \mu \mathrm{l}$ (data not shown). These concentrations comprise DNA of M. tuberculosis as well as DNA from host cells and commensal organisms present in sputum samples.

\subsection{Detection of M. tuberculosis in clinical samples stored in PS-MTM}

Of the 297 specimens prospectively collected; 41 were excluded from routine diagnostics due to poor sputum quality in $8(2.7 \%)$ or culture contamination in $33(11.1 \%)$ of which M. tuberculosis was detected in 5. Thus, from the specimens analyzed $(n=256), 26(10.2 \%)$ were culture positive including $13(5.1 \%)$ that were smear positive. Real-time PCR of sputum in PS-MTM was positive in all smear-positive specimens (13/13) and 7/13 (54\%) of smear-negative culturepositive sputum specimens (Tab. 5). PCR was positive in another 8 specimens $(3.1 \%)$ that were negative by both microscopy and culture.

Table 5: Laboratory performance of real-time PCR detection of M. tuberculosis from sputum specimens (n=256) inoculated in PrimeStore ${ }^{\circledR}$ - Molecular Transport Medium (PS-MTM) using the swabbing procedure compared to routine smear microscopy and culture

\begin{tabular}{|c|c|c|c|}
\hline & \multicolumn{2}{|c|}{ Culture positive $(n=26)$} & \multirow{2}{*}{$\begin{array}{l}\text { Culture negative } \\
\qquad(\mathbf{n}=\mathbf{2 3 0})\end{array}$} \\
\hline & $\begin{array}{c}\text { Smear-positive } \\
\qquad(\mathrm{n}=13)\end{array}$ & $\begin{array}{c}\text { Smear-negative } \\
(n=13)\end{array}$ & \\
\hline PCR positive & $13(100 \%)$ & $7(54 \%)$ & $8(3.5 \%)$ \\
\hline PCR negative & 0 & $6(46 \%)$ & $222(96.5 \%)$ \\
\hline
\end{tabular}




\section{DISCUSSION}

This laboratory evaluation demonstrates that PS-MTM rapidly inactivates $M$. tuberculosis in sputum specimens thereby rendering the specimen safe, compatible with various nucleic acid extraction systems and suitable for downstream molecular processing in a routine diagnostic setting. These observations are in line with two studies describing evaluation of this transport medium for molecular processing of sputum specimens for respiratory viruses (Daum, et al., 2011, Schlaudecker et al., 2014). Our observations are of importance as concentrations of viral DNA or RNA are generally higher than of M. tuberculosis DNA in sputum samples.

Inactivation of the organism was measured by the ability to grow in an internationally standardized culture medium; the current gold standard to viability testing (van Zyl-Smit et al., 2011). Maximum bacillary load of $M$. tuberculosis in a clinical sputum specimen is approximately $10^{6}$ organisms $/ \mathrm{ml}$ (Rieder et al., 2007); therefore we performed most laboratory experiments at this concentration and a 100-fold higher. With regard to mycobacterial inactivation, M. tuberculosis in pure culture, grown to a concentration of $10^{8} \mathrm{CFU} / \mathrm{ml}$, was inactivated within 5 seconds of exposure to the PS-MTM transport medium. However, time to inactivation of $M$. tuberculosis was considerably longer in the proteinaceous matrix of sputum. The sputum to PS-MTM ratio is important and should be at least 1:2 to achieve complete inactivation in a reasonable period of time (60 minutes; 30 minutes at 1:3 ratio) at $M$. tuberculosis concentration of $1.5 \times 10^{8} \mathrm{CFU} / \mathrm{ml}$. The rapid inactivation is in line with findings from Daum et al. (2014), which showed that PS-MTM is an effective medium to inactivate a pure culture of $M$. tuberculosis at $\sim 10^{6} \mathrm{CFU} / \mathrm{ml}$ at an exposure time of 30 minutes. This is a similar effect as achieved by the Xpert SR buffer where an 8 and $9 \log$ reduction was demonstrated at 15 minutes and 2 hours respectively (Helb, et al., 2010). A minimum time of 60 minutes from collection to specimen processing is likely to be routine and will provide a safe specimen for downstream testing, particularly in resource-constrained settings.

Stability of M. tuberculosis DNA in PS-MTM at ambient temperature was demonstrated for a period of 4 weeks. This period is more than sufficient to use PS-MTM in a diagnostic approach. However, additional follow-up testing is warranted to determine the value of PS-MTM for longterm bio banking. A limitation of this evaluation was not directly comparing the effect on DNA stability in sputum at ambient temperature in comparison to the PS-MTM sample. 
PS-MTM was compatible with the most commonly used DNA extraction systems and in most cases did not differ in their ability to detect positives. Thus it could be used in most laboratories, regardless of their set-up, and for a variety of downstream molecular applications. The two automated magnetic bead-based extraction systems (NucliSENS easyMAG and MagNA Pure system), ideal for routine services due to their robustness, performed best and demonstrated similar high performance, efficiency and reproducibility. The observed difference in performance between magnetic bead and silica extraction systems may be due to loss of DNA released by PS-MTM, particularly during processing prior to silica binding.

Evaluation of almost 300 clinical sputum specimens using PS-MTM as pre-step demonstrated good sensitivity and specificity compared to smear microscopy and liquid culture. Performance was in line with generally accepted characteristics of molecular testing for M. tuberculosis: 90$100 \%$ sensitivity in microscopy smear-positive sputum specimens and 55-75\% among smearnegative cases (Armand et al., 2011, Dinnes et al., 2007, Miller et al., 2011, Steingart et al., 2014). There was no apparent effect of chemical composition of PS-MTM on detection of lower bacillary loads as diagnostic performance among smear-negative cases was adequate.We did not test amplification suppression, but think that the effect, if present, was minimal as we were able to successfully amplify $M$. tuberculosis at relatively low $\mathrm{CFU} / \mathrm{ml}$ as well as detect smear negative TB cases in the clinical laboratory evaluation. This was achieved by transferring a very small quantity of the primary sputum specimen $(0.2 \mathrm{ml})$ into PS-MTM. Although the volume captured on a swab is only an estimated fifth of the volume required for the Xpert assay, this small volume was sufficient to adequately detect $M$. tuberculosis at sensitivities similar to that of the Xpert assay.

The method of using swabs to inoculate sputum into PS-MTM provides an adequate yield for molecular processing. Moreover, it provides a practical approach that allows healthcare workers to directly inoculate sputum into PS-MTM for rapid inactivation and stabilization after the specimen is produced. Such an alternative method is warranted as pipetting sputum at healthcare facilities would not be feasible. 


\section{CONCLUSIONS}

PS-MTM may have several applications in addition to the diagnosis of M. tuberculosis including: safe transport and preservation of specimen for molecular testing, its inactivating capability of pure cultures could allow the convenient ambient temperature transport of the organism for surveillance activities (i.e. molecular typing and sequencing) and ambient temperature molecular bio banking which could remove the current storage costs associated with the conventional method. Continuous improvements in molecular diagnostics require innovative methods for transporting specimens safely, efficiently and without compromising integrity that may influence diagnosis. With regard to $M$. tuberculosis detection, PS-MTM provides a promising tool for transport between clinical and centralized diagnostic facilities for molecular testing which could expand molecular diagnosis of tuberculosis in resource-poor settings.

\section{Acknowledgements}

We would like to thank the Department of Medical Microbiology, University of Pretoria for funding the research and Longhorn Vaccines and Diagnostics, San Antonio, Texas, USA for providing the PrimeStore Molecular Transport Medium. We further thank the National Health Laboratory Service, Tshwane Academic Division for proving strains and allowing use of laboratory facilities.

\section{References:}

Armand, S., Vanhuls, P., Delcroix, G., Courcol, R., Lemaitre, N., 2011. Comparison of the Xpert MTB/RIF test with an IS6110-TaqMan real-time PCR assay for direct detection of Mycobacterium tuberculosis in respiratory and nonrespiratory specimens. J Clin Microbiol. 49, 1772-1776. 10.1128/JCM.02157-10

Banada, P.P., Sivasubramani, S.K., Blakemore, R., Boehme, C., Perkins, M.D., Fennelly, K., Alland, D., 2010. Containment of bioaerosol infection risk by the Xpert MTB/RIF assay and its applicability to point-of-care settings. J Clin Microbiol. 48, 3551-3557. 10.1128/JCM.01053-10 
Banda, H., Harries, A., Boeree, M., Nyirenda, T., Banerjee, A., Salaniponi, F., 2000. Viability of stored sputum specimens for smear microscopy and culture [Notes from the Field]. The International Journal of Tuberculosis and Lung Disease. 4, 272-274.

Bollela, V.R., Sato, D.N., Fonseca, B.A., 1999. McFarland nephelometer as a simple method to estimate the sensitivity of the polymerase chain reaction using Mycobacterium tuberculosis as a research tool. Braz J Med Biol Res. 32, 1073-1076.

Daum, L.T., Choi, Y., Worthy, S.A., Rodriguez, J.D., Chambers, J.P., Fischer, G.W., 2014. A molecular transport medium for collection, inactivation, transport, and detection of Mycobacterium tuberculosis. Int J Tuberc Lung Dis. 18, 847-849. 10.5588/ijtld.13.0021

Daum, L.T., Ismail, N., Fourie, P.B., Hoosen, A.A., Omar, S.V., Worthy, S.A., Fisher, G.W., 2011. A Rapid, Collection-to-Detection PCR System for the Universal Detection of $M$. Tuberculosis, 29th Annual Meeting of the European Society for Paediatric Infectious Diseases, The Hague, The Netherlands.

Daum, L.T., Worthy, S.A., Yim, K.C., Nogueras, M., Schuman, R.F., Choi, Y.W., Fischer, G.W., 2011. A clinical specimen collection and transport medium for molecular diagnostic and genomic applications. Epidemiol Infect. 139, 1764-1773. 10.1017/S0950268810002384

Dinnes, J., Deeks, J., Kunst, H., Gibson, A., Cummins, E., Waugh, N., Drobniewski, F., Lalvani, A., 2007. A systematic review of rapid diagnostic tests for the detection of tuberculosis infection. Health Technol Assess. 11, 1-196.

Djelouagji, Z., Drancourt, M., 2006. Inactivation of cultured Mycobacterium tuberculosis organisms prior to DNA extraction. J Clin Microbiol. 44, 1594-1595. 10.1128/JCM.44.4.15941595.2006

Doig, C., Seagar, A.L., Watt, B., Forbes, K.J., 2002. The efficacy of the heat killing of Mycobacterium tuberculosis. J Clin Pathol. 55, 778-779. 10.1136/jcp.55.10.778

Global Laboratory Initiative, 2014. Mycobacteriology Laboratory Manual, WHO Press, Geneva, Switzeland, pp. 154.

Hasan, M.R., Tan, R., Al-Rawahi, G.N., Thomas, E., Tilley, P., 2012. Short-term stability of pathogen-specific nucleic acid targets in clinical samples. J Clin Microbiol. 50, 4147-4150. 10.1128/JCM.02659-12

Helb, D., Jones, M., Story, E., Boehme, C., Wallace, E., Ho, K., Kop, J., Owens, M.R., Rodgers, R., Banada, P., Safi, H., Blakemore, R., Lan, N.T., Jones-Lopez, E.C., Levi, M., Burday, M., Ayakaka, I., Mugerwa, R.D., McMillan, B., Winn-Deen, E., Christel, L., Dailey, P., Perkins, M.D., Persing, D.H., Alland, D., 2010. Rapid detection of Mycobacterium tuberculosis and rifampin resistance by use of on-demand, near-patient technology. J Clin Microbiol. 48, 229-237. 10.1128/JCM.01463-09

Ingersoll, J., Bythwood, T., Abdul-Ali, D., Wingood, G.M., Diclemente, R.J., Caliendo, A.M., 2008. Stability of Trichomonas vaginalis DNA in Urine Specimens. J Clin Microbiol. 46, 16281630. 10.1128/jcm.02486-07 
Inoue, S., Becker, A.L., Kim, J.H., Shu, Z., Soelberg, S.D., Weigel, K.M., Hiraiwa, M., Cairns, A., Lee, H.B., Furlong, C.E., Oh, K., Lee, K.H., Gao, D., Chung, J.H., Cangelosi, G.A., 2014. Semi-automated, occupationally safe immunofluorescence microtip sensor for rapid detection of Mycobacterium cells in sputum. PLoS One. 9, e86018. 10.1371/journal.pone.0086018

Miller, M.B., Popowitch, E.B., Backlund, M.G., Ager, E.P., 2011. Performance of Xpert MTB/RIF RUO assay and IS6110 real-time PCR for Mycobacterium tuberculosis detection in clinical samples. J Clin Microbiol. 49, 3458-3462. 10.1128/JCM.05212-11

Murray, P.R., Baron, E.J., 2007. Manual of clinical microbiology, ASM Press, Washington, D.C.

Paramasivan, C.N., Narayana, A.S., Prabhakar, R., Rajagopal, M.S., Somasundaram, P.R., Tripathy, S.P., 1983. Effect of storage of sputum specimens at room temperature on smear and culture results. Tubercle. 64, 119-124. 10.1016/0041-3879(83)90036-3

Paulson, T., 2013. Epidemiology: A mortal foe. Nature. 502, S2-S3. 10.1038/502S2a

Rieder, H.L., Van Deun, A., Kam, K.M., Kim, S.J., Chonde, T.M., Trebucq, A., Urbanczik, R., 2007. Priorities for Tuberculosis Bacteriology Services in Low-Income Countries, International Union Against Tuberculosis and Lung Disease, France.

Schlaudecker, E.P., Heck, J.P., MacIntyre, E.T., Martinez, R., Dodd, C.N., McNeal, M.M., Staat, M.A., Heck, J.E., Steinhoff, M.C., 2014. Comparison of a new transport medium with universal transport medium at a tropical field site. Diagn Microbiol Infect Dis. 80, 107-110. 10.1016/j.diagmicrobio.2014.05.018

Siddiqi, S.H., Rusch-Gerdes, S., 2006. MGIT Procedure Manual For BACTEC MGIT 960 TB System, Foundation for Innovative New Diagnostics, Geneva, Switzeland.

Steingart, K.R., Schiller, I., Horne, D.J., Pai, M., Boehme, C.C., Dendukuri, N., 2014. Xpert(R) MTB/RIF assay for pulmonary tuberculosis and rifampicin resistance in adults. Cochrane Database Syst Rev. 1, CD009593. 10.1002/14651858.CD009593.pub3

van Zyl-Smit, R.N., Binder, A., Meldau, R., Mishra, H., Semple, P.L., Theron, G., Peter, J., Whitelaw, A., Sharma, S.K., Warren, R., Bateman, E.D., Dheda, K., 2011. Comparison of quantitative techniques including Xpert MTB/RIF to evaluate mycobacterial burden. PLoS One. 6, e28815. 10.1371/journal.pone.0028815

WHO, 2010. Global Tuberculosis Control, WHO Press, Geneva, Switzeland.

WHO, 2013. Automated real-time nucleic acid amplification technology for rapid and simultaneous detection of tuberculosis and rifampicin resistance: Xpert MTB/RIF system for the diagnosis of pulmonary and extrapulmonary TB in adults and children (Policy Update), WHO Press, Geneva, Switzeland.

Winn Jr, W.C., Allen, S.D., Janda, W.M., 2005. Introduction to Microbiology. In: Koneman's Color Atlas and Textbook of Diagnostic Microbiology Lippincott Williams \& Wilkins. 